\title{
Valor pronóstico de la hipertrofia ventricular izquierda
}

Vakili BA, Okin PM, Devereaux RB.Prognostic implications of left ventricular hypertrophy. Am Heart J 2001;141:334-41.

\section{Objetivo}

Evaluación de la asociación entre hipertrofia ventricular izquierda (HVI) en condiciones basales y eventos clínicos adversos futuros.

\section{Fuente y selección de datos}

Revisión no sistemática de MEDLINE y otras fuentes de publicaciones desde enero de 1960 a enero de 2000 que relacionaran datos de HVI identificada por electrocardiograma (ECG) o ecocardiografía en condiciones basales y desarrollo de morbilidad cardiovascular o mortalidad de cualquier causa.Se utilizaron riesgos relativos (RR) ajustados por factores de riesgo mayores.

\section{Pacientes}

Se identificaron 20 estudios que abarcaban un total de 48.545 individuos con adecuada descripción electrocardiográfica o ecocardiográfica de la HVI y datos de seguimiento concernientes a la morbilidad cardiovascular y mortalidad de cualquier causa.

\section{Resultados principales}

HVI evaluada por ECG: en 6 estudios (5 prospectivos y uno retrospectivo) con 14.450 pacientes que incluían sujetos con infarto, hipertensión (HTA), ancianos, la cohorte de Framingham y adultos blancos con HTA (edad media en los estudios fue $\geq 49$ años, prevalencia de HVI 6-35\%).La HVI estuvo asociada con 1,6 a 4 veces más riesgo de eventos cardíacos futuros. En un ensayo
Clínico, 8 estudios de cohorte y un estudio retrospectivo que incluían 38.262 pacientes con HTA, pacientes sometidos a angiografía, con angina, infarto, la población de Framingham, ancianos o pacientes con enfermedad coronaria documentada (edad media en los estudios $\geq 48$ años, prevalencia de HVI $5 \%$ a $44 \%$ ), el RR para mortalidad de cualquier causa fue de 1,5 a 6,8 veces mayor entre aquellos con HVI.

Hipertrofia parietal evaluada por ecocardiografía: en 3 estudios prospectivos que incluyeron 3.651 pacientes con HTA o pertenecientes a la cohorte de Framingham (edad media $\geq 43$ años, prevalencia de HVI 16-44\%), el RR asociado con morbilidad cardiovascular fue de 1.5 a 3.5 veces mayor en aquellos con HVI. En 7 estudios prospectivos que incluían 5.478 pacientes de la población de Framingham, con insuficiencia renal terminal, afroamericanos sometidos a angiografía e hipertensos el RR asociado con mortalidad de cualquier causa fue de 1.0 a 8.0.

EI RR ajustado global para morbilidad cardiovascular y mortalidad de cualquier causa en pacientes con HVI en los estudios que utilizaron criterios electrocardiográficos o ecocardiográficos combinados fue de 2.3 y 2.5 respectivamente.

\section{Conclusión}

La HVI predice un alto riesgo independientemente de los factores de riesgo mayores. Estos resultados establecen una fuerte relación entre la HVI y la evolución adversa futura y enfatizan la importancia clínica de su detección.

\section{Comentario}

La HVI es, inicialmente, una respuesta al incremento de la poscarga ventricular asociada a una elevada resistencia vascular sistémica y puede ser vista en cierto modo como una respuesta necesaria y protectora. EI ECG ha sido utilizado históricamente para su reconocimiento siendo hoy en día acompañado en forma más significativa por la ecocardiografía dada la mayor sensibilidad del método. Este último, permite además realizar la medición de la masa ventricular izquierda y la identificación de las diferentes patentes de hipertrofia que agregan información pronóstica adicional.1

Ha sido evidente en la mayoría de los estudios que la presencia de HVI supone un mayor riesgo de eventos cardiovasculares, que incluyen morbimortalidad por insuficiencia cardíaca, fibrilación auricular y muerte súbita en pacientes hipertensos.2

La revisión actual se enfrenta con la dificultad habitual que implica tratar de unificar la información proveniente de estudios con diseño diferente, con distinta prevalencia de enfermedad, diferente criterio diagnóstico utilizado para definir HVI y clasificación de eventos realizada en forma no sistemática. Estos problemas, junto con la distinta definición del punto final (en el caso de morbilidad cardiovascular) y un seguimiento a veces no suficientemente largo (incluyendo estudios con seguimiento de sólo 48 hs) debilitan las conclusiones. Sin embargo, a favor de su validez externa * y en concordancia con la mayoría de los estudios, aporta interesante información en donde se observa que independientemente de la población evaluada la presencia de HVI es un marcador pronóstico independiente una vez ajustado por los factores de riesgo tradi- cionales. Las razones detrás de esta robusta asociación no son del todo claras pero parecen estar en relación a un compromiso de la función sisto-diastólica, una mayor predisposición de los pacientes con HVI a desarrollar arritmias y a un posible empeoramiento de la isquemia miocárdica.

En base a estos datos la prevención y quizás la reversión de la HVI secundaria a HTA es un objetivo deseable. Esto ha sido observado utilizando tratamientos farmacológicos y no farmacológicos, con una mejoría en el corto plazo en la función sistólica (si esta afectada), en la función diastólica y un aumento en la capacidad de ejercicio. ${ }^{3}$ Un interesante meta-análisis que incluyó 39 estudios clínicos aleatorizados, controlados, con diseño dobleciego de pacientes tratados por HTA encontró una mayor regresión de la HVI mediante una reducción más marcada de la presión arterial y una mayor duración de la terapia (13\% con IECA, $9 \%$ con bloqueantes cálcicos, $6 \%$ con beta-bloqueantes y $7 \%$ con diuréticos). ${ }^{4}$ En este sentido, el reciente estudio HOPE que evaluó pacientes con alto riesgo cardiovascular, encontró una reducción significativa del riesgo de muerte, IAM, ACV e insuficiencia cardiaca en relación con la disminución del desarrollo o regresión de la HVI con el uso de ramipril. 5

Estos resultados enfatizan la necesidad de establecer como objetivos claves la prevención del desarrollo de la hipertrofia parietal así como también su regresión una vez que esta se ha establecido en la población de pacientes hipertensos no complicados basados en un adecuado y estricto control de la HTA.

\section{Dr. Pablo F. Oberti [ Servicio de Cardiología.Hospital Italiano de Buenos Aires ]}

\footnotetext{
Referencias

1. Koren MJ, Devereaux RB, Casale PN, Savage DD, Laragh JH.Relation of left ventricular mass and geometry to morbidity and mortality in uncomplicated essential hypertension.Ann Intern Med 1991;114:345-352.

2.Lip GYH, Felmeden DC, Li-Saw-Hee FL, et al.Hypertensive heart disease:a complex syndrome or a hypertensive "cardiomyopathy"? Eur Heart J.2000;21:1653-1665. 3.Lorell BH, Carabello BA.Left ventricular hypertrophy.Pathogenesis, detection and prognosis.Circulation 2000;102:470-479.

4.Schmieder RE, Martus P, Klingbeil A.Reversal of left ventricular hypertrophy in essential hypertension:a meta-analysis of randomized double-blind studies. JAMA 1996:275:1507-1513. 5.Mathew J, Sleight P, Lonn E, et al; for the Heart Outcomes Prevention Evaluation (HOPE) Investigators. Reduction of cardiovascular risk by regression of electrocardiographic markers of left ventricular hypertrophy by the angiotensin-converting enzyme inhibitor ramipril.Circulation 2001:104:1615-1621.
} 